Niemann, H. B. \& Owen, T. C. Icarus 171, 153-170 (2004).

10.Guillot, T., Stevenson, D. J., Hubbard, W. B. \& Saumon, D. in Jupiter: The Planet, Satellites and Magnetosphere (eds Bagenal, F., Dowling, T. E. \& McKinnon, W. B.) 35-57 (Cambridge Univ.
Press, 2004)

11.Mordasini, C., Alibert, Y., Klahr, H. \& Henning, T. Astron. Astrophys. 547, A111 (2012).

12.Paardekooper, S.-J. \& Mellema, G. Astron. Astrophys.

425, L9-L12 (2004).

13. Moll, R., Garaud, P., Mankovich, C. \& Fortney, J. J.
Astrophys. J. 849, 24 (2017).

14.Vazan, A., Helled, R. \& Guillot, T. Astron. Astrophys. 610, L14 (2018).

15. Moutou, C. et al. Icarus 226, 1625-1634 (2013).

16. Thorngren, D. P., Fortney, J. J., Murray-Clay, R. A. \&

Lopez, E. D. Astrophys. J. 831, 64 (2016).

\title{
No bacteria found in healthy placentas
}

\section{Analysis of hundreds of placentas provides convincing evidence that this organ does not harbour microorganisms that can enter the fetal gut - a key finding for research into how the human microbiota is established. SEE ARTICLE P.329}

\section{NICOLA SEGATA}

$\mathrm{T}$ The early human embryo is free of microorganisms, whereas the postweaning infant hosts a community of microbes - a microbiota - comparable in complexity to that in adults. How and when the symbiosis between a human and their microbiota is established are subjects of active research. On page 329 , de Goffau et al. ${ }^{1}$ provide evidence that the placenta, which acts as the interface between the maternal body and the fetus, is not colonized by microorganisms in healthy pregnancies and is thus unlikely to be the main gateway for the development of the infant microbiota in utero.

If the microbial colonization of humans occurs in the womb, then this would have key implications for the shaping of the early immune system. An infant's first stool is already populated with microorganisms, but it is unclear whether this is solely the result of microbial acquisition during ${ }^{2}$ and $\mathrm{after}^{3}$ delivery, or if microbes also reach and colonize the fetus before birth. Because sampling fetal gut content is much more difficult than collecting the placenta and amniotic fluid during (elective) caesarean delivery, scientists have focused on the latter two at the interface between the maternal and fetal bodies. The conclusive identification of microbial communities in and on the placenta would indeed suggest that microbes colonize the fetus, but, in the past few years, evidence has been presented both that supports ${ }^{4-7}$ and that refutes ${ }^{8-11}$ the long-standing dogma that the placenta and amniotic fluid are sterile in physiological conditions - that is, during healthy pregnancy. The debate about this issue therefore remains open $^{12,13}$ (Fig. 1).

It is not disputed that, during a healthy pregnancy, the placenta and amniotic fluid cannot host a concentration of bacteria as high as that observed in the adult mouth or gut. The technical challenge in studies of placenta samples is therefore to distinguish any microorganisms that are truly present in small quantities on these tissues from those found on laboratory tools and from contamination of the samples during collection. Small amounts of microbial contamination can be pervasive, and sources range from the air to supposedly sterile DNA-extraction kits ${ }^{14}$ and other items associated with DNA processing and sequencing ${ }^{15}$. There was thus a need for studies to rigorously account for potential contamination; these studies would also need a sufficiently large sample size to ensure statistical robustness. De Goffau and colleagues now report on such a study.

The authors analysed placenta samples from 537 women - by far the largest number of samples used in a study of this kind - using a thorough DNA-sequencing approach to search for microbial content. They used the same DNA-extraction toolkit and sequencing procedures on negative controls - 'blank' samples that were supposedly free from biological material. They also used positive controls, produced by spiking placental samples with a known amount of the bacterium Salmonella bongori, to calibrate the abundance of other microbes that might be in the sample. The sequencing was performed using two complementary techniques, known as shotgun metagenomics ${ }^{16}$ and $16 \mathrm{~S}$ rRNA gene amplicon sequencing ${ }^{17}$, to account for technique-specific potential biases. The results were clear: the placenta does not harbour microbes during healthy pregnancy, and contamination issues were a convincing explanation for the presence of any detected bacteria.

Some of the details reported in the paper reveal how pervasive contaminating microbes can be when concentrations of bacteria in the samples are very low. For example, two potential

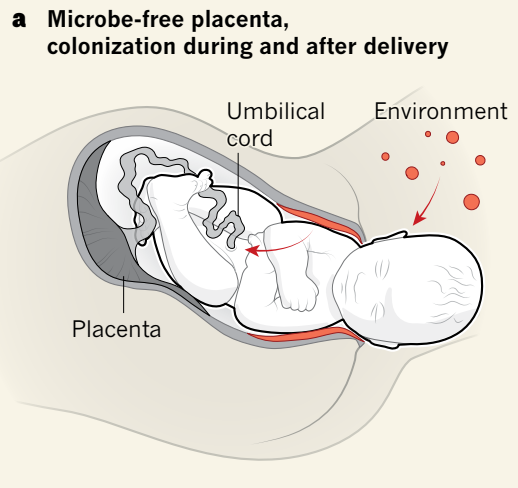

b In utero colonization from placenta

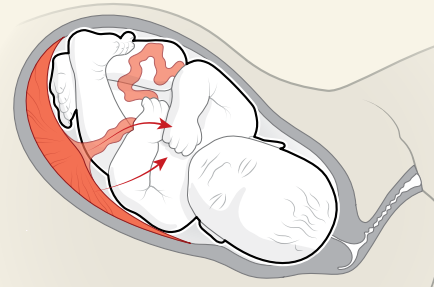

C Microbe-free placenta, in utero colonization

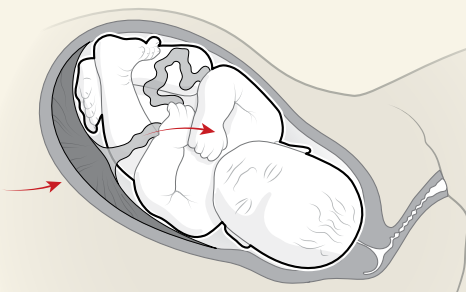

Figure 1 | Scenarios for bacterial colonization of the infant gut. a, It has long been thought that the human placenta and the fetus are free of microorganisms. Newborns were therefore expected to acquire gut bacteria from the mother during delivery and from the environment (red regions indicate sources of bacteria), with further influences associated with the mode of delivery and feeding regime (breastfeeding or formula milk). b. However, in the past few years, evidence has been published ${ }^{8-11}$ suggesting that the placenta contains bacteria and that bacterial colonization of the fetal gut therefore occurs in the womb. c, In utero colonization of the fetal gut from the mother might also occur under certain circumstances, even if the placenta is microbe-free. De Goffau et al. ${ }^{1}$ now report convincing evidence that the placenta is free of bacteria during healthy pregnancies, thus ruling out the scenario in $\mathbf{b}$. 
disease-causing bacteria,Vibrio cholerae and Streptococcus pneumoniae, were detected by shotgun metagenomics and matched strains of bacteria that had previously been sequenced on the same apparatus. The detection of these bacteria is therefore most probably the result of cross-contamination of the authors' sequencing machine. The ability of modern sequencing methods to detect low numbers of bacteria is thus a problem in some experiments, because even tiny levels of contaminants can result in a false-positive detection. Greater contamination of the authors' samples occurred during the earlier stages of sample preparation than in later stages. The authors confirmed previous reports ${ }^{14}$ stating that a relatively rich microbiota was present in commercial DNA-extraction kits, and identified company-specific communities of bacteria from the genetic material extracted from the blank control samples.

Overall, the complex procedures used by de Goffau and colleagues to identify contaminants allowed them to reach a clear conclusion: only one type of bacterium was convincingly found in the placental samples in their study, and it was in only about $5 \%$ of those samples. This finding provides strong evidence that there is no functional microbiota in the placenta and suggests that it is highly unlikely that infants acquire microbes from the placenta in normal physiological conditions.

The bacterium occasionally detected in the placenta was Streptococcus agalactiae. If present in the mother during childbirth, $S$. agalactiae can be transmitted to the newborn and cause pneumonia, septicaemia and meningitis; several clinical practices are used to prevent such transmission ${ }^{18}$. The identification of S. agalactiae in some of the placenta samples in the study does not conflict with the dogma that the womb is microbe-free in healthy pregnancies, because this bacterium is associated with disease. Indeed, the finding that $S$. agalactiae is the only bacterium to be found on the placenta, and in a low number of samples, mirrors the expectation that a small fraction of pregnant mothers are infected with it, and that it can undergo intrauterine transmission - therefore adding credibility to the experimental findings.

De Goffau and colleagues' carefully controlled, large-scale study was needed to provide strong evidence for the absence of bacteria in the placenta. As such, the study also sets a benchmark for investigations dealing with other human organs or tissues that, at most, carry a small number of bacteria, such as the lungs or blood. Nevertheless, negative results are hard to prove conclusively, so the dogma that the womb is free of microbes should be further investigated. Bacteria can overcome many host barriers under certain conditions, and just one bacterial cell that reaches the gut of the fetus could potentially start in utero colonization. How the symbiosis of a human host with their microbiota is established remains an intriguing, fundamental question, but we can now be confident that the placenta is not a microbial reservoir and therefore is not a major direct stream of diverse microbes to the fetus under healthy conditions.

Nicola Segata is in Department CIBIO,

University of Trento, Trento 38123, Italy.

e-mail:nicola.segata@unitn.it

1. de Goffau, M. C. et al. Nature 572, 329-334 (2019).

2. Ferretti, P. et al. Cell Host Microbe 24, 133-145 (2018).

3. Korpela, K. et al. Genome Res. 28, 561-568 (2018).

4. Leon, L. J. et al. Appl. Environ. Microbiol. 84, e00483-18 (2018).

5. Leiby, J. S. et al. Microbiome 6, 196 (2018).

6. Theis, K. R. et al. Am. J. Obstet. Gynecol. 220, 267-267 (2019)

7. Lim, E. S., Rodriguez, C. \& Holtz, L. R. Microbiome 6, 87 (2018)
8. Collado, M. C., Rautava, S., Aakko, J., Isolauri, E. \& Salminen, S. Sci. Rep. 6, 23129 (2016).

9. Urushiyama, D. et al. Sci. Rep. 7, 12171 (2017).

10.Aagaard, K. et al. Sci. Transl. Med. 6, 237 ra65 (2014).

11.Antony, K. M. et al. Am. J. Obstet. Gynecol. 212, 653 (2015).

12. Perez-Muñoz, M. E. Arrieta, M. C. Ramer-Tait, A. E. \& Walter, J. Microbiome 5, 48 (2017).

13.Bushman, F. D. Am. J. Obstet. Gynecol. 220 213-214 (2019).

14.Salter, S. J. et al. BMC Biol. 12, 87 (2014)

15. Eisenhofer, R. et al. Trends Microbiol. 27, 105-117 (2019).

16.Quince, C., Walker, A. W., Simpson, J. T., Loman, N. J. \& Segata, N. Nature Biotechnol. 35 833-844 (2017).

17. Hamady, M. \& Knight, R. Genome Res. 19, 1141-1152 (2009).

18.Johri, A. K. et al. Nature Rev. Microbiol. 4, 932-942 (2006).

This article was published online on 31 July 2019.

\section{CELL BIOLOGY}

\section{How plants perceive salt}

High salt levels in the soil harm plant growth and limit crop yields. A salt-binding membrane lipid has been identified as being essential for salt perception and for triggering calcium signals that lead to salt tolerance. SEE ARTICLE P.341

\section{LEONIE STEINHORST \& JÖRG KUDLA}

$\mathrm{S}_{\mathrm{s}}^{\mathrm{s}}$ alt as a nutrient for humans is a doubleedged sword, being tasty in small amounts but generating an adverse response as the concentration rises. Distinct protein receptors have been shown to mediate these opposing reactions in animals. Excessive uptake of salt is not only unhealthy for humans but also detrimental for plants, because high levels of salt in the soil limit plant growth and crop yields. This is of concern, given that such conditions affect approximately $7 \%$ of land globally, including areas used for agriculture, and high salinity affects about $30 \%$ of irrigated crops ${ }^{1}$. On page 341 , Jiang et al. ${ }^{2}$ shed light on how plants recognize salt in their surroundings.

The salt sodium chloride $(\mathrm{NaCl})$ is the main cause of salt stress in plants. It is toxic to cells because at high intracellular concentrations, $\mathrm{Na}^{+}$ions compete with other ions for involvement in biological reactions. It also has a negative effect on cellular functions by perturbing the balance of ions and thus of water - generating what is called an osmotic perturbation. It was not known how plants perceive stress generated by high salt and whether they can distinguish between ionic and osmotic perturbations.

The exposure of plants to salt stress triggers an immediate temporally and spatially defined rise in the concentration of cytoplasmic calcium ions $\left(\mathrm{Ca}^{2+}\right)$. It is thought that a calcium channel, of as yet unknown identity, provides a

route for $\mathrm{Ca}^{2+}$ to enter cells during such calcium signalling. This $\mathrm{Ca}^{2+}$ signal leads to cellular adaption to salt stress in plant roots, and the subsequent formation of $\mathrm{Ca}^{2+}$ waves that spread over long distances and mediate adaptation responses throughout the entire plant ${ }^{3,4}$. Central to salt tolerance is the evolutionarily conserved SOS pathway. In this pathway, proteins such as SOS3, which can bind $\mathrm{Ca}^{2+}$ ions, decode the $\mathrm{Ca}^{2+}$ signal and activate ${ }^{5}$ a protein kinase enzyme called SOS2. This enzyme, in turn, activates a

"It was not

known how plants perceive stress generated by high salt." protein in the cell membrane called SOS 1 , which is a type of protein known as an antiporter that can transport $\mathrm{Na}^{+}$ions out of the cell. SOS2 also promotes the sequestration of $\mathrm{Na}^{+}$from the cytoplasm into an organelle called a vacuole ${ }^{6}$. However, the components and mechanisms governing the perception of extracellular $\mathrm{Na}^{+}$ and driving salt-induced $\mathrm{Ca}^{2+}$ signalling were unknown.

Jiang and colleagues performed a genetic screen using the model plant Arabidopsis thaliana to identify mutant plants that had an abnormally low $\mathrm{Ca}^{2+}$-signalling response to high $\mathrm{Na}^{+}$exposure, but that could still generate $\mathrm{Ca}^{2+}$ signals when challenged with other types of stress. Taking this approach, they identified a plant that had a mutation 